\title{
Estado nutricional en la marginación y la pobreza de adultos triquis del estado de Oaxaca, México
}

\author{
Rosa María Ramos Rodríguez ${ }^{1}$ y Karla Sandoval Mendoza ${ }^{2}$
}

Forma de citar

Ramos Rodríguez RM, Sandoval Mendoza K. Estado nutricional en la marginación y la pobreza de adultos triquis del estado de Oaxaca, México. Rev Panam Salud Pública. 2007;22(4):260-7.

RESUMEN Objetivos. Estudiar los posibles cambios seculares en el estado nutricional de sujetos del grupo étnico triqui del estado de Oaxaca, México, para profundizar el conocimiento del proceso descrito como transición nutricional.

Métodos. A partir de los datos antropométricos de tres estudios realizados anteriormente en este grupo —a fines del siglo XIX, en 1940 y en 2002-, se calcularon los promedios y las puntuaciones $\mathrm{z}$ de la estatura, del peso y del indice de masa corporal (IMC) de adultos triquis, empleando los referentes de Frisancho. Los resultados numéricos se analizaron estadísticamente mediante las pruebas de la $t$ de Student y la $\chi^{2}$. La información sociocultural se obtuvo a través de entrevistas y de observación directa, así como de fuentes bibliográficas.

Resultados. La estatura de los triquis se ha mantenido muy baja. Se encontró un mayor número de mujeres de estatura pequeña o muy pequeña. Esto, sumado a la menor escolaridad y el mayor grado de analfabetismo y de monolingüismo de las mujeres, refleja una actitud cultural que no favorece al género femenino. Resulta preocupante la aparición de casos de sobrepeso y obesidad entre los hombres estudiados en 2002 con respecto a los incluidos en el estudio de 1940; en 2002 también las mujeres presentaron sobrepeso.

Conclusiones. A pesar de las limitaciones de todo estudio retrospectivo, los resultados obtenidos confirman que actualmente el estado nutricional de los triquis es diferente al de hace 60 años, en relación con los cambios culturales que está viviendo este grupo étnico. Para profundizar el conocimiento de este fenómeno epidemiológico en el que coexisten la desnutrición y la obesidad, es preciso realizar estudios transdisciplinarios que consideren la interacción de componentes biológicos, sociales y culturales interpretados en perspectiva sistémica, para adoptar medidas integrales teniendo presente las particularidades bioculturales de cada grupo humano.

Palabras clave Transición nutricional, sobrepeso, obesidad, pobreza, población indígena, México.

1 Instituto de Investigaciones Antropológicas, Universidad Nacional Autónoma de México, México D.F., México. La correspondencia debe dirigirse a Rosa María Ramos Rodríguez, Instituto de Investigaciones Antropológicas, Circuito exterior s/n, Ciudad Universitaria, CP 04510, México D.F., México; tel.: +52 (55) 5675 0270; fax: +52 (55) 5622 9651; correo electrónico: rmrr@servidor.unam.mx

2 Unidad de Biología Evolutiva, Universidad Pompeu Fabra, Barcelona, España.
Actualmente se reconoce que la obesidad es un problema mundial de salud (1) que se ha venido gestando en los últimos 60 años, durante los cuales se han producido grandes cambios sociales, económicos y tecnológicos que han alterado los patrones de vida en prácticamente todo el planeta. Si bien se pensaba que era privativa de los países desarrollados o de los grupos humanos con alto poder adquisitivo, actualmente se está incrementando en 
los países en vías de desarrollo y se observa tanto en poblaciones urbanas como en rurales (2).

Ante esta situación, resulta importante profundizar el conocimiento de este fenómeno epidemiológico, identificando su presencia y su evolución en diferentes contextos económicos y culturales. En efecto, en la mayoría de los trabajos se proporciona información derivada de encuestas de cobertura nacional, con representación estatal, como en el caso de México (3), pero se pierden las particularidades de los contextos sociales y culturales específicos en los que viven los diversos grupos humanos, las cuales son muy importantes para identificar las diferentes circunstancias que motivan la obesidad.

Como ocurre en muchos países de Latinoamérica, esta clase de información es escasa y aún más lo es aquella necesaria para conocer la evolución de la prevalencia de los distintos estados nutricionales en poblaciones marginadas. Sin embargo, rescatando los datos derivados de estudios anteriores, incluso cuando se llevaron a cabo con otros propósitos, es posible sacar conclusiones sobre dicho proceso. Partiendo de esta propuesta, se consideró de interés estudiar a los triquis, un grupo étnico mexicano con una cultura propia. Debido a una situación socioeconómica de alta marginación, la desnutrición parece inevitable en este grupo, pero no la obesidad.

Los triquis se localizan en la región Mixteca alta y baja, en el estado de Oaxaca de la República Mexicana. Su territorio se distribuye entre los distritos políticos de Juxtlahuaca, Tlaxiaco y Putla (figura 1). Los grupos asentados en la Mixteca alta tienen como cabecera municipal la localidad de San Andrés Chicahuaxtla, mientras que los de la Mixteca baja tienen como cabecera San Juan Copala, en el municipio de Santiago Juxtlahuaca, en las inmediaciones de las montañas de Putla y Juxtlahuaca.

Se trata de una comunidad de aproximadamente 15448 miembros (4) con una identidad étnica muy arraigada. Este arraigo se manifiesta a través de su organización política y social tradicional $(5,6)$, adaptada estratégica-
FIGURA 1. Situación geográfica del municipio de Juxtlahuaca, estado de Oaxaca, México

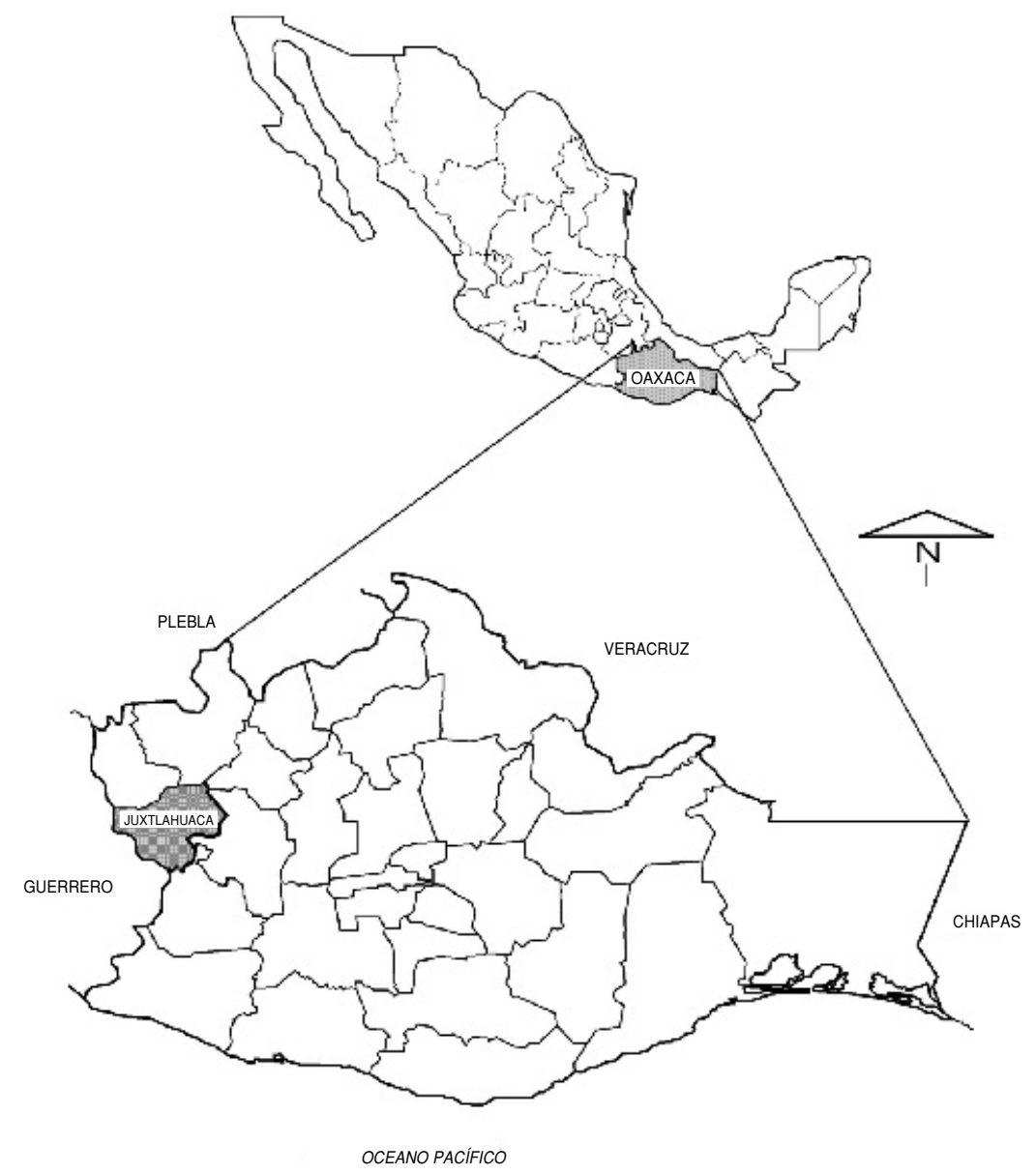

mente a las transformaciones de un entorno político, social y económico cambiante $(4,7)$. Al respecto, para los fines de este trabajo, es pertinente recalcar que los triquis aún tratan de conservar los patrones de reproducción endogámica propios de su cultura (8). Sin embargo, diversas circunstancias políticas, económicas y sociales presentes en México como en el ámbito internacional han motivado la migración de un alto porcentaje de miembros de este grupo, dentro del país $(4,7)$, a la Ciudad de México o a las ciudades norteñas de agricultura intensiva comercial, o bien hacia los Estados Unidos de Norteamérica $(9,10)$. Esto ha provocado, entre otras causas, la modificación de algunos patrones de identidad étnica en las generaciones más jóvenes de migrantes, que incorporan en sus familias y en la comunidad nuevos elementos culturales y sociales que forman parte de su vida cotidiana $(4,6$, 11). Teniendo presentes estas modificaciones, se consideró relevante estudiar su posible repercusión en el estado nutricional de los adultos triquis, a partir de la información derivada de tres estudios somatométricos realizados en este grupo étnico en diferentes épocas.

\section{MATERIALES Y MÉTODOS}

El presente estudio se apoya en los valores de peso y estatura de los sujetos estudiados en 1940 por Comas et al. (12) y en el año 2002 por Sandoval (13). Se calcularon los promedios y las desviaciones estándar de estas variables. Se emplearon como datos com- 
plementarios los valores medios de estatura y peso registrados a fines del siglo XIX por Frederick Starr (14).

Se calculó en cada caso el índice de la masa corporal (IMC) (peso [kg]/ estatura $\left.^{2}\left[\mathrm{~m}^{2}\right]\right)$. Para clasificar a los individuos en función de su estado nutricional (peso insuficiente, peso normal, sobrepeso u obesidad) según el IMC, se tomaron como límites los valores que propone la Organización Mundial de la Salud (15) (peso insuficiente: IMC < 18,5; peso normal: 18,5 $\leq$ IMC $<$ 25,0; sobrepeso: 25,0 $\geq$ IMC < 30,0; obesidad: IMC $\geq 30,0)$. La estatura de los sujetos se clasificó según los intervalos indicados por Comas (16) (hombres: muy grande, superior a $1,80 \mathrm{~m}$; grande, entre 1,70 y 1,79 m; mediana, entre 1,60 y 1,69 m; pequeña, entre 1,50 y $1,59 \mathrm{~m}$; muy pequeña, inferior a 1,49 m; mujeres: muy grande, superior a 1,68 m; grande, entre 1,59 y 1,67 m; mediana, entre 1,49 y $1,58 \mathrm{~m}$; pequeña, entre 1,40 y $1,48 \mathrm{~m}$; muy pequeña, inferior a $1,39 \mathrm{~m}$ ).

Se calcularon las puntuaciones $z$ de la estatura, según la edad y el sexo, utilizando como referentes los estándares antropométricos proporcionados por Frisancho (17). Las diferencias entre las muestras de 1940 y de 2002 se analizaron estadísticamente a través de las pruebas de la $t$ de Student y de la $\chi^{2}$ para distinguir la asociación entre variables.

A partir de los datos recabados en las entrevistas, las observaciones realizadas durante el trabajo en la comunidad y la información derivada de diversas fuentes bibliográficas, fue posible identificar los aspectos culturales más relevantes de este grupo étnico.

Se presentan a continuación las muestras consideradas en el estudio, así como algunas de sus características.

\section{Los triquis de Chicahuaxtla, Oaxaca, a finales del siglo XIX}

Con el objetivo de caracterizar a las poblaciones del sur de México, a finales del siglo XIX Starr realizó una expedición por el sureste mexicano. En Oaxaca, uno de los estados que visitó, midió a 99 hombres y 25 mujeres tri- quis residentes en Chicahuaxtla. En el trabajo que publicó en 1902 notificó, entre otros datos, los promedios de la estatura de estos sujetos (14).

\section{Los triquis de San Andrés Chicahuaxtla en 1940}

En el marco de un programa general de investigaciones elaborado por el Instituto Nacional de Antropología e Historia para estudiar los distintos grupos indígenas mexicanos, entre 1940 y 1941 se llevaron a cabo en la región triqui, en especial entre los habitantes de San Andrés Chicahuaxtla y de rancherías aledañas, estudios de corte etnográfico, lingüístico y somatométrico. Este último estudio se realizó bajo la conducción de Juan Comas, para determinar las características físicas del grupo (12). La muestra estudiada estuvo compuesta por 101 hombres adultos, normales, sin padecimientos aparentes, de entre 20 y 45 años.

Tanto Comas (18) como Basauri (19), responsable de los trabajos etnográficos, refirieron que en aquella época, las poblaciones triquis sólo eran accesibles a través de caminos de herradura y de veredas transitables a pie, y que la base de su economía familiar era la agricultura y, eventualmente, el comercio y el trabajo a jornal. Estos autores constataron que prácticamente todos los indígenas de San Andrés hablaban sólo la lengua triqui, a excepción de cuatro de ellos que también hablaban español. Aunque en aquel entonces había dos escuelas en San Andrés Chicahuaxtla, la asistencia de los niños triquis era sumamente reducida. Basauri, al describir la impresión general que tuvo de estos indígenas, los definió como "gente en extremo miserable" y aseguró que se encontraban pésimamente alimentados y que la desnutrición y el raquitismo eran frecuentes entre los niños (19).

\section{Los triquis de San Juan Copala en 2002}

Entre los años 2000 y 2002, una de las autoras de este trabajó realizó el proyecto "Antropología molecular del grupo indígena trique" en San Juan Copala, Oaxaca (13). En el marco de este proyecto, y a petición de los sujetos que colaboraban en el estudio, en enero de 2002 se efectuó un estudio antropométrico en el que se registraron el peso y la estatura de 173 individuos (49 hombres y 124 mujeres) originarios del municipio de Juxtlahuaca, Oaxaca, de edades comprendidas entre 15 y 79 años.

En 1990, el Consejo Nacional de Población había clasificado a Juxtlahuaca entre los municipios mexicanos con grado de marginación alto (20). En el momento del estudio, las condiciones de vida que prevalecían en San Juan Copala denotaban importantes atrasos de desarrollo. En esta región se seguía hablando el triqui, pero en la muestra estudiada, el $83 \%$ de los hombres hablaba también español; en contraste, sólo el 47,5\% de las mujeres era bilingüe. A su vez, el nivel de escolaridad era muy bajo y el analfabetismo, elevado ( $47,30 \%$ del total), sobre todo entre las mujeres $(56,9 \%$, frente a $21,7 \%$ entre los hombres).

Por otra parte, la infraestructura de servicios básicos seguía siendo muy deficiente, pues si bien la energía eléctrica llegaba a la mayoría de las viviendas, no todas contaban con agua potable y no había alcantarillado en la localidad. Por último, puede mencionarse que existía una carretera que facilitaba la comunicación con el resto del estado, pero el transporte público era escaso e irregular.

\section{RESULTADOS}

En el cuadro 1 se presentan el número de casos, los valores mínimo y máximo, la media y la desviación estándar de la estatura, el peso y el IMC - cuando se contó con la información para calcularlo- de los sujetos incluidos en los tres estudios mencionados. No se apreciaron diferencias entre la estatura media de los hombres y las mujeres de las tres muestras. Al aplicar la prueba de la $t$ de Student a los datos de los hombres estudiados en 1940 y en 2002, no se encontraron dife- 
CUADRO 1. Estatura, peso e índice de la masa corporal (IMC) de adultos triquis del estado de Oaxaca, México, 1901 (14), 1940 (12) y 2002 (13)

\begin{tabular}{|c|c|c|c|c|c|c|c|}
\hline & \multicolumn{3}{|c|}{ Estatura $(\mathrm{m})$} & \multicolumn{2}{|c|}{ Peso (kg) } & \multicolumn{2}{|c|}{ IMC } \\
\hline & 1901 & $1940^{\mathrm{a}}$ & 2002 & $1940^{a}$ & 2002 & $1940^{a}$ & 2002 \\
\hline \multicolumn{8}{|l|}{ Hombres } \\
\hline$n$ & 99 & 101 & 49 & 101 & 49 & 101 & 49 \\
\hline Mínimo & 1,35 & 1,46 & 1,40 & 42,00 & 40,00 & 17,56 & 18,77 \\
\hline Máximo & 1,68 & 1,67 & 1,69 & 65,00 & 82,20 & 24,83 & 30,94 \\
\hline Media & 1,55 & 1,56 & 1,57 & 50,84 & 59,95 & 20,75 & 24,21 \\
\hline $\mathrm{DE}^{\mathrm{b}}$ & & 0,04 & 0,07 & 4,88 & 9,56 & 1,47 & 2,83 \\
\hline Diferencia entre medias & 0,01 & 9,11 & 3,46 & & & & \\
\hline$(\text { IC95\%) })^{c}$ & & \multirow{2}{*}{\multicolumn{2}{|c|}{$\begin{array}{c}(-0,01-0,02) \\
\quad>0,05^{\mathrm{e}}\end{array}$}} & \multirow{2}{*}{\multicolumn{2}{|c|}{$\begin{array}{c}(6,78-11,43) \\
<0,0001\end{array}$}} & \multirow{2}{*}{\multicolumn{2}{|c|}{$\begin{array}{c}(2,75-4,15) \\
<0,0001\end{array}$}} \\
\hline$P^{\mathrm{d}}$ & & & & & & & \\
\hline \multicolumn{8}{|l|}{ Mujeres } \\
\hline$n$ & 25 & $\ldots$ & 124 & $\cdots$ & 124 & $\ldots$ & 124 \\
\hline Mínimo & 1,32 & $\cdots$ & 1,30 & $\ldots$ & 36,00 & $\ldots$ & 19,29 \\
\hline Máximo & 1,56 & $\ldots$ & 1,57 & $\cdots$ & 88,00 & $\ldots$ & 41,28 \\
\hline Media & 1,43 & $\ldots$ & 1,45 & $\ldots$ & 53,71 & $\ldots$ & 25,60 \\
\hline $\mathrm{DE}^{\mathrm{b}}$ & $\ldots$ & $\ldots$ & 0,05 & $\ldots$ & 8,63 & $\ldots$ & 3,80 \\
\hline
\end{tabular}

a Valores calculados a partir de los datos de Comas et al. (12).

b DE: desviación estándar.

IC95\%: intervalo de confianza de 95\%.

d Prueba de la $t$ de Student.

e Diferencia no significativa.

CUADRO 2. Frecuencia y proporción de casos según la estatura y el sexo entre adultos triquis de San Juan Copala, estado de Oaxaca, México, 2002 (13)

\begin{tabular}{lcccc}
\hline \multicolumn{1}{c}{ Estatura } & $\begin{array}{c}\text { Hombres }[\mathrm{H}] \\
n \text { (proporción) }\end{array}$ & $\begin{array}{c}\text { Mujeres [M] } \\
n \text { (proporción) }\end{array}$ & $\begin{array}{c}\text { Diferencia entre } \\
\text { proporciones [H - M] } \\
(\text { IC95\%) }\end{array}$ & $P^{\mathrm{b}}$ \\
\hline Media & $18(0,367)$ & $24(0,193)$ & $\begin{array}{c}0,174 \\
(0,022-0,326)\end{array}$ & $<0,05$ \\
Pequeña & $24(0,490)$ & $86(0,694)$ & $\begin{array}{c}-0,204 \\
(-0,366--0,042)\end{array}$ & $<0,05$ \\
Muy pequeña & $7(0,143)$ & $14(0,113)$ & $\begin{array}{c}0,030 \\
(-0,083-0,143)\end{array}$ & $>0,05^{d}$ \\
\multicolumn{1}{c}{ Total } & $49(1,000)$ & $124(1,000)$ & & \\
\hline
\end{tabular}

a Categorías propuestas por Comas (16):

Hombres: muy grande (> 1,80 m), grande $(1,70-1,79 \mathrm{~m})$, mediana $(1,60-1,69 \mathrm{~m})$, pequeña $(1,50-1,59 \mathrm{~m})$, muy pequeña $(<1,49 \mathrm{~m})$. Mujeres: muy grande $(>1,68 \mathrm{~m})$, grande $(1,59-1,67 \mathrm{~m})$, mediana $(1,49-1,58 \mathrm{~m})$, pequeña $(1,40-1,48 \mathrm{~m})$, muy pequeña $(<1,39 \mathrm{~m})$.

b IC95\%: intervalo de confianza de $95 \%$

${ }^{c}$ Prueba de la $x^{2}$.

d Diferencia no significativa.

rencias significativas entre las medias de esta dimensión corporal (cuadro 1). Cabe señalar que la mayoría de las personas estudiadas en 2002 se clasificaron en la categoría de estatura pequeña y que la proporción de mujeres en esta categoría o en la de estatura muy pequeña era mayor que la de hombres (80,7\% frente a $63,3 \%$; prueba de la $\chi^{2}, P>0,05$ ) (cuadro 2).

Para ahondar en la caracterización del crecimiento cefalocaudal alcanzado, se calcularon las puntuaciones $z$ de la estatura de los individuos estudiados en 1940 y en 2002. Como no se conocía la edad de los hombres inclui- dos en el estudio de 1940 - ya que únicamente se había indicado que tenían entre 25 y 45 años-, se utilizaron para el cálculo los valores correspondientes al intervalo de 30,0-34,9 años de edad $(176,5 \pm 6,8 \mathrm{~cm})$ según el referente empleado. De acuerdo con la distribución de las puntuaciones $z$, sólo el 5,0\% de los hombres medidos en 1940 y el 9,7\% de las mujeres estudiadas en 2002 se encontraron entre $\pm 1,5$ desviaciones estándar (DE), intervalo considerado como normalidad estadística. En contraste, un mayor número de hombres del grupo medido en 2002 se ubicó en este intervalo (25\%) (figura 2).

Por otra parte, según la prueba de la $t$ de Student, se observaron diferencias significativas entre los promedios del peso y del IMC de los hombres estudiados en 1940 y en 2002 (cuadro 1), con valores mayores en estos últimos. En los adultos, el peso corporal relacionado con la edad es un mal indicador del estado nutricional, pero el IMC aporta buena información relacionada con el componente graso del cuerpo. Según la clasificación basada en el IMC, mientras en 1940 casi la totalidad de los hombres estudiados se situaron dentro del rango de normalidad, el $34,7 \%$ de los individuos de la muestra estudiada en 2002 presentaba sobrepeso u obesidad. Debe destacarse que en 2002, la proporción de sobrepeso y obesidad fue mayor en las mujeres $(50,8 \%)$ (cuadro 3) y resultó similar a la hallada a escala nacional (21).

\section{DISCUSIÓN}

La comunicación con los triquis asentados en la Mixteca es difícil, tanto por su ubicación geográfica como por la poca confianza que les inspiran las personas ajenas a su comunidad y la violencia en la que están inmersos $(4,22)$. Esto supone limitaciones para realizar estudios en la región. En consecuencia, la información disponible sobre este grupo étnico es insuficiente, sobre todo en lo relativo a la salud. Por esta razón, resultó útil emplear los datos somatométricos de investigaciones anteriores para realizar nuevas interpretaciones, a pesar de las limita- 
FIGURA 2. Distribución de la frecuencia de casos según la puntuación $z$ de la estatura de adultos triquis, estado de Oaxaca, México, 1941 (12) y 2002 (13)

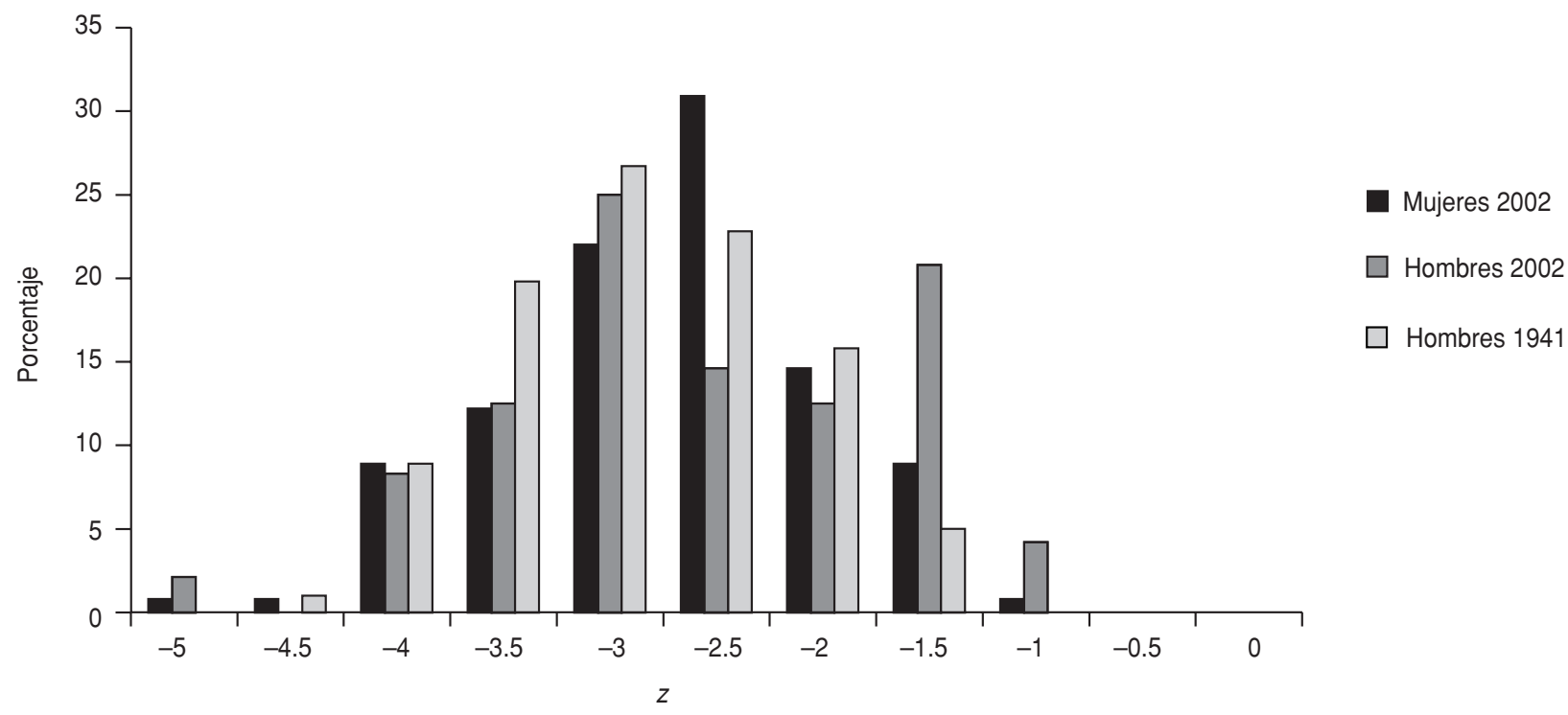

CUADRO 3. Frecuencia de casos clasificados según el índice de masa corporal (IMC) entre adultos triquis de San Juan Copala, estado de Oaxaca, México, 1940 (12) y 2002 (13)

\begin{tabular}{|c|c|c|c|}
\hline \multirow{3}{*}{$\frac{\text { Estado nutricional según el IMC }}{\text { Peso insuficiente }}$} & \multirow{2}{*}{$\begin{array}{c}1940 \\
\text { Hombres } \\
n(\%)\end{array}$} & \multicolumn{2}{|c|}{2002} \\
\hline & & $\begin{array}{c}\text { Hombres } \\
n(\%)\end{array}$ & $\begin{array}{c}\text { Mujeres } \\
n(\%)\end{array}$ \\
\hline & $6 \quad(5,9)$ & $(0,0)$ & $(0,0)$ \\
\hline Peso normal & $95 \quad(94,1)$ & $32 \quad(65,3)$ & $61 \quad(49,2)$ \\
\hline Sobrepeso u obesidad & $0 \quad(0,0)$ & $17 \quad(34,7)$ & $63 \quad(50,8)$ \\
\hline Total & $101(100,0)$ & $49(100,0)$ & $124(100,0)$ \\
\hline
\end{tabular}

${ }^{a}$ Categorías propuestas por la OMS (peso insuficiente: IMC $<18,5$; peso normal: $18,5 \leq$ IMC $<25,0$; sobrepeso: $25,0 \leq$ IMC $<30,0$; obesidad: IMC $\geq 30,0)(15)$

ciones que conlleva toda metodología retrospectiva.

Los resultados obtenidos a partir de las comparaciones del peso, la estatura y el IMC confirman que el estado nutricional de los hombres triquis recientemente estudiados es diferente al de hace 60 años. Este fenómeno no pudo constatarse en las mujeres, puesto que no se cuenta con información anterior.

A pesar de las limitaciones impuestas por la falta de información se pueden hacer diversos comentarios. Por un lado, es preocupante que desde fines del siglo XIX hasta la fecha, la estatura de los triquis no haya variado y se mantenga en valores muy bajos, a diferencia de lo ocurrido en otras poblaciones $(23,24)$. Se conoce la estrecha vinculación que guardan el crecimiento y el desarrollo físico de los menores con los determinantes sociales que conforman el entorno en el que viven $(25,26)$. Así, teniendo en cuenta las precarias condiciones de vida de la población triqui, inferidas a partir de estudios anteriores $(14,18,19)$, y conociendo el pobre desarrollo de San Juan Copala constatado en 2002 (13), puede concluirse que la baja estatura de los sujetos y su insignificante modificación a lo largo de los años se deben, muy probablemente, a las restricciones que el entorno les ha impuesto generación tras generación. Esto ha propiciado la desnutrición durante los primeros años de la vida, lo cual ha limitado el crecimiento y el desarrollo físico. Esta situación lamentablemente se corrobora con la información proporcionada por el segundo censo nacional de talla en niños de primer grado de primaria, realizado en México en 1994 (27), y el estudio del índice de riesgo nutricional estimado por municipio (28). Según estas fuentes, en Santiago Juxtlahuaca, la prevalencia media de baja estatura para la edad en niños de entre seis y nueve años fue del $59 \%$ y la puntuación $z$ media, de $-2,17$ (27); además, en este municipio, el riesgo de desnutrición en los primeros años de la vida es muy alto (28).

Resulta más preocupante que, conforme a los datos del estudio realizado en 2002, las mujeres presentan mayor probabilidad de tener una estatura pequeña, lo que supone un riesgo para la gestación. Esta situación, junto con la menor escolaridad, y el mayor analfabetismo y monolingüismo de las muje- 
res, traduce una actitud cultural que no favorece al género femenino.

Cabe mencionar que a pesar de que en la literatura se reconoce una relativa ventaja biológica femenina que aparentemente hace a las mujeres más resistentes a los agentes agresores del entorno (29), por razones sociales y culturales, éstas pueden volverse más vulnerables ante el medio físico y social hostil en el que viven. En efecto, se han descrito situaciones en las que se otorga diferente valor simbólico al individuo según el sexo, lo cual ocasiona que durante los primeros años de la vida, con frecuencia, los hombres reciban mayor atención, alimentos de mejor calidad y mayor asistencia médica en caso de enfermedad (30).

Si bien prácticamente no se han observado modificaciones en la estatura de los adultos triquis, en la actualidad es alarmante la presencia de sobrepeso u obesidad. Aun tratándose de una población con alta marginalidad, el cuadro epidemiológico actual presenta la tendencia que se viene observando en naciones en vías de desarrollo (31). A pesar de que los sujetos estudiados en 1940 habían sido desnutridos en los primeros años de su vida, de adultos sólo el 6\% presentaba bajo peso, y ninguno, sobrepeso u obesidad, contrariamente a lo observado en 2002.

Diversas hipótesis tratan de explicar la epidemia de obesidad (2). Algunas de ellas asocian la condición de desnutrición en los primeros años de la vida y los cambios metabólicos que se gestan por esta situación y propician alteraciones en la vida adulta (32). La "teoría del gen ahorrador" de Neel, reformulada en 1998 (33), propone un enfoque más integral en el que destaca la importancia de los estilos de vida, centrándose en la dieta y la actividad física desarrollada. Otras propuestas se refieren al tipo de dieta adoptada y a su vinculación con la situación económica del país y de las familias (34, 35). Popkin ha propuesto una teoría con un enfoque más global, conocida como "transición nutricional", en la que se centra la atención en los rápidos cambios de la dieta vinculados con modificaciones de la actividad física y su repercusión en la composición cor- poral, a la luz de las transformaciones demográficas y socioeconómicas que están teniendo lugar a partir del reordenamiento político y económico en el contexto de la globalización $(36,37)$.

En un trabajo anterior, a través del estudio del sistema sanguíneo $\mathrm{ABO}$ en la muestra de San Juan Copala, se corroboró un alto componente amerindio, con un bajo grado de mestizaje con población de origen europeo (99,3\% de alelo O y $0,66 \%$ de heterocigosidad de este sistema) (13), lo cual resulta congruente con las prácticas endogámicas que prevalecen en este grupo étnico. Por lo tanto, es poco probable que la composición genética de la población triqui haya cambiado de manera importante, lo que permite suponer que la predisposición genética a la obesidad puede haber estado presente en esta población, pero sólo en la actualidad se han identificado casos de sobrepeso u obesidad. Respecto a posibles modificaciones relacionadas con la actividad física, hay que tener en cuenta que las condiciones de vida de los triquis siguen siendo precarias, a pesar de las mejoras acaecidas entre 1940 y 2002; así, para lograr la subsistencia (6), continúan siendo necesarias tareas que demandan un gasto energético importante, aunque algunas de ellas implican en la actualidad menor trabajo físico. Por ejemplo, los triquis han diversificado sus actividades económicas (comercio, jornal agrícola, albañilería); ahora tienen la posibilidad de utilizar molinos eléctricos para elaborar la masa de maíz, en lugar de hacerlo manualmente (11), o de utilizar transporte público motorizado para desplazarse a otras comunidades para comprar o vender sus productos, en vez de hacerlo a pie, como en el pasado.

El sobrepeso y la obesidad pueden deberse a un efecto epigénico en el que se vinculan tanto el peculiar metabolismo asociado con la condición de desnutrición en los primeros años de la vida (32) como el seguimiento de una dieta desequilibrada, rica en hidratos de carbono simples y grasas. $\mathrm{Al}$ respecto, durante el trabajo de campo realizado en San Juan Copala, se observó que, a pesar del fuerte arraigo de sus prácticas culturales, los triquis han añadido a su dieta tradicional basada principalmente en maíz, frijoles y pimientos $(5,6)$ - alimentos densamente calóricos, especialmente refrescos embotellados y harinas industrializadas de bajo precio $(11,13)$. No obstante, dado su carácter retrospectivo, el presente estudio no cuenta con información detallada relativa a las prácticas alimentarias del grupo. Sin embargo, teniendo en cuenta los cambios en los patrones de vida y de consumo ocurridos tanto en el entorno nacional como en el internacional, en el contexto del proceso de globalización, y su influencia inevitable a escala local, puede inferirse que, como ha ocurrido en otras poblaciones en México $(34,38)$, la dieta de los triquis ha cambiado paulatinamente y los productos densamente calóricos se han convertido en un componente importante de su alimentación cotidiana. A escala individual, diversas circunstancias socioculturales pueden explicar esta evolución, entre las que cabe mencionar la posible conveniencia de adquirir alimentos de bajo costo para satisfacer la sensación de saciedad aunada al placer gustativo que proporcionan estos alimentos (35), a la facilidad para procurárselos y prepararlos, y a una propaganda excesiva por parte de las empresas que los fabrican y comercializan (39).

Además del prestigio que los triquis dicen adquirir al consumir este tipo de alimentos (11), es importante tener presente que este pueblo considera bellas a las mujeres que no son delgadas "porque representan buena salud" (13).

Son muchas las explicaciones posibles del fenómeno epidemiológico de desnutrición y obesidad, que es una realidad que no se puede evadir. Sin embargo, para encontrar respuestas es necesario aceptar que se trata de una realidad compleja. El presente trabajo invita a realizar investigaciones transdisciplinarias, que permitan comprender este fenómeno en su perspectiva sistémica (40) y adoptar medidas integrales que consideren, de manera especial, las particularidades bioculturales de cada grupo humano. 


\section{REFERENCIAS}

1. Popkin BM, Doak CM. The obesity epidemia is a worldwide phenomenon. Nutr Rev. 1998; 56:106-14.

2. Méndez MA, Monteiro CA, Popkin B. Overweight exceeds underweight among women in most developing countries. Am J Clin Nutr. 2005;81:714-21.

3. Olaiz-Fernández G, Rivera-Dommarco J, Shamah-Levy T, Rojas R, VillalpandoHernández S, Hernández-Ávila M, et al. Encuesta Nacional de Salud y Nutrición 2006. Cuernavaca, México: Instituto Nacional de Salud Pública; 2006.

4. Lewin P. La gente de la lengua completa (yi ni' nanj ni' ïnj). El grupo etnolingüístico triqui. En: Barabas A, Bartolomé MA, coord. Configuraciones étnicas en Oaxaca. Perspectiva etnográfica para las autonomías. Vol. II: Mesoetnias. México: Instituto Nacional de Antropología e Historia e Instituto Nacional Indigenista; 1999. Pp. 215-65.

5. Huerta-Ríos C. Organización socio-política de una minoría nacional. Los triquis de Oaxaca. México: Instituto Nacional Indigenista; 1981.

6. García-Alcaraz A. Tinujei: Los triquis de Copala. 2. ${ }^{\mathrm{a}}$ ed. México: Centro de Investigaciones y Estudios Superiores en Antropología Social; 1997.

7. Bartolomé MA. La tierra de la diversidad: Relaciones interétnicas y procesos identitarios en Oaxaca. En: Bartolomé MA, coord. Vol. II: Visiones de la diversidad. Relaciones interétnicas e identidades indígenas en el México actual. México: Instituto Nacional de Antropología e Historia; 2005. Pp. 61-139.

8. Hollenbach EE. El parentesco entre los triques de Copala, Oaxaca. América Indígena 1973; 33(1):167-86.

9. Velasco Ortiz L. Migración, género y etnicidad: mujeres indígenas en la frontera de Baja California y California. Rev Mex Soc. 2000;62: 145-71.

10. Vargas Becerra PN, Flores Dávila JI. Los indígenas en ciudades de México: el caso de los mazahuas, otomíes, triquis, zapotecos y mayas. Papeles de Población. 2002;34:235-57.

11. Mendoza González Z. De la casa del nene al árbol de las placentas. Proceso reproductivo, saberes y transformación cultural entre los triquis de Copala en la Merced. [tesis doctoral]. México: Centro de Investigaciones y Estudios Superiores en Antropología Social, 2004.

12. Comas J, Faulhaber J. Somatometría de los indios triques de Oaxaca, México. México: Instituto de Investigaciones Históricas, Universidad Nacional Autónoma de México; 1965.

13. Sandoval Mendoza K. Aspectos teóricos y prácticos de la antropología molecular, el caso del grupo trique. [tesis de licenciatura]. México: Escuela Nacional de Antropología e Historia, 2004.

14. Starr F. The physical characters of the indians of Southern Mexico. The Decennial Publica- tions, University of Chicago; 1902, vol. 4:53109.

15. Kaufer M, Tavano L, Ávila H. Obesidad en el adulto. En: Casanueva E, Kaufer M, Pérez A, Arroyo P, eds. Nutriología Médica. México: Fondo Nestlé para la Salud/Editorial Médica Panamericana; 2001. Pp. 283-310.

16. Comas J. Manual de Antropología Física. $2^{\text {a }}$ edición. México: Instituto de Investigaciones Antropológicas, Universidad Nacional Autónoma de México; 1976

17. Frisancho R. Anthropometric standards for the assessment of growth and nutrition status. Ann Arbor: The University of Michigan Press; 1990.

18. Comas J. El problema social de los indios triques en Oaxaca. América indígena. 1942; 2(1):51-7.

19. Basauri C. Los indios triques de Oaxaca. En: La población indígena de México. Tomo II. Etnografía. México: Secretaría de Educación Pública; 1940. Pp. 427-63.

20. México, Consejo Nacional de Población, Comisión Nacional del Agua. Indicadores socioeconómicos e índice de marginación municipal, 1990. México: Consejo Nacional de Población y Comisión Nacional del Agua; 1993.

21. Olaiz G, Rojas R, Barquera S, Shamah T, Aguilar C, Cravioto P et al. Encuesta Nacional de Salud, 2000. Tomo 2. La Salud de los adultos. Cuernavaca, México: Instituto Nacional de Salud Pública, Secretaría de Salud; 2003.

22. Parra Mora LJ, Hernández Díaz J. Violencia y cambio social en la región Trique. México: Consejo Estatal de la Población de Oaxaca y Universidad Autónoma Benito Juárez de Oaxaca; 1994.

23. Ramos-Galván R. Análisis de dos estudios de peso y talla hechos con 50 años de diferencia en niños de la ciudad de México. Bol Med Hosp Infant México 1978;34:441-63.

24. Bogin B, Loucky J. Plasticity, political economy, and physical growth status of Guatemala Maya children living in the United States. Am J Phys Anthropol. 1997;102:17-32.

25. Martorell R, Habicht JP. Growth in early childhood in developing countries. En: Falkner F, Tanner JM, eds. Vol 2. Human growth A comprehensive treaties. 5. ${ }^{\text {a }}$ ed. New York: Plenum Press; 1986. Pp. 241-62.

26. Waterlow JC. Malnutrición proteico-energética. Washington, D.C.: OPS; 1996. (Publicación científica 555).

27. México. Sistema Nacional para el Desarrollo Integral de la Familia. Segundo censo nacional de talla en niños de primer grado de primaria. México: SEP, DIF, CONAFE, OPS, UNICEF; 1996.

28. Roldán A, Ávila A, Chávez A, Álvarez M, Muñoz M, Shamah T. Regionalización de la situación nutricional en México a través de un índice de riesgo nutricional. México: Instituto Nacional de Ciencias Médicas y Nutrición
Salvador Zubirán, Sociedad Latinoamericana de Nutrición; 2004

29. Stinson S. Sex differences in environmental sensitivity during growth and development. Yearbook of Physical Anthropology. 1985;28: $123-47$.

30. Das-Gupta M. Selective discrimination against female children in rural Punjab, India. Pop Devel Rev. 1987;13:77-100.

31. Popkin BM. The nutrition transition and obesity in the developing world, Symposium: Obesity in developing countries: Biological and ecological factors. J. Nutr. 2001;131(3): 871s-73s

32. Sawaya AL, Martins PA, Grillo LP, Florêncio TT. Long-term effects of early malnutrition on body weight regulation. Nut Rev. 2004;62: S127-33.

33. Neel J. The "thrifty genotype" in 1998. Nut Rev. 1999;57(5) (part II): S2-S9.

34. Arroyo P, Loria A, Méndez O. Changes in the household calorie supply during the 1994 economic crisis in Mexico and its implications on the obesity epidemia. Nut Rev. 2004;62(7): s163-68.

35. Drewnowski A, Specter SE. Poverty and obesity: the role of energy density and energy costs. Am J Clin Nutr. 2004;76:6-16.

36. Popkin BM. The nutrition transition and its health implications in lower income countries. Public Health Nutr. 1998;1(1):5-21.

37. Popkin BM. The nutrition transition: an overview of patterns of change. Nut Rev. 2004;62 (7):S140-3.

38. Barquera S, Rivera J, Espinosa-Montero J, Safdie M, Campirano F, Monterrubio E. Energy and nutrient consumption in Mexican women 12-49 years of age: Analysis of the National Nutrition Survey 1999. Salud Publica Mex 2003:45:S530-39.

39. Popkin B. Technology, transport, globalization and the nutrition transition food policy. Food Policy. 2006;31:554-69.

40. García R. Sistemas complejos. Conceptos, método y fundamentación epistemológica de la investigación interdisciplinaria. Barcelona: Editorial Gedisa; 2006.

Manuscrito recibido el 29 de agosto de 2006. Aceptado para publicación, tras revisión, el 7 de julio de 2007. 
ABSTRACT Objectives. To further understanding of the nutritional transition process by studying possible changes over time in the nutritional status of the Triqui ethnic group in the state of Oaxaca, Mexico.

\section{Nutritional status of the poor, marginalized adults of the Triqui ethnic group in Oaxaca, Mexico}

Methods. A retrospective study was conducted using anthropometric data available from three prior studies (end of the 19th century, 1940, and 2002). Means and $z$ scores for height, weight, and body mass index of Triqui adults were calculated using Frisancho reference data. Statistical analyses of the results were performed with Student's $t$ and chi-square tests. Sociocultural information was collected through interviews and direct observations, as well as from the available literature.

Results. The height of Triqui adults has remained very low. A majority of Triqui women were found to be short or very short; this, coupled with the low level of education and high rates of illiteracy and monolingualism, is indicative of a culture that does not favor females. The appearance of overweight and obesity among the men studied in 2002, relative to those studied in 1940, is cause for concern. Overweight was also recorded among the women in 2002.

Conclusions. Despite the limitations shared by all retrospective studies, these results confirm that the nutritional status of the Triquis is different now from what it was 60 years ago and that these changes are related to the cultural changes the group is experiencing. To further understand this epidemiologic phenomenon, where malnutrition and obesity coexist, cross-disciplinary studies are needed to analyze the biological, social, and cultural factors involved, so that culturally-appropriate measures can be adopted.

Key words Nutrition phenomena, nutritional status, overweight, obesity, ethnic groups, Mexico.
Nutrition and an Active Life: From Knowledge to Action is an anthology by leading public health experts from $\mathrm{PAHO}$ and the international development community. The book's selections focus on how research in nutrition and the promotion of active lifestyles can provide vital input for the creation of public policy and planning and for the design, implementation, monitoring, and evaluation of programs.

This publication is an important contribution that should be of particular interest to practitioners, researchers, and decisionmakers in the fields of health promotion, community education, nutrition, maternal and child health, physical activity, policy development in public health and urban planning, and other related areas.

To order this publication: http://publications.paho.org; Fax: (301) 2069789; E-mail: paho@pmds.com; PAHO/WHO office in your country

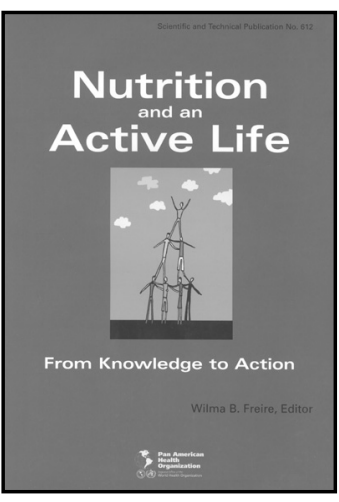

2005, pp., 260

ISBN: 9275116121

US\$ 30.00 in Latin America and the Caribbean/

US\$20.00 elsewhere

Order code: SP 612 